\title{
New Medications for the Treatment of Diabetes
}

\author{
Satish K. Garg, Dominique Giordano, Tyler Gallo, and Viral N. Shah
}

\section{Introduction}

With THE RISING PREVAlENCE OF Diabetes WORLDWIDE, there has been an increasing interest in finding new ways to manage diabetes. In this chapter, we are going to review abstracts related to newer medications that are being used or that are under investigation for the management of diabetes. We reviewed hundreds of manuscripts/abstracts indexed in PubMed from July 2015 to June 2016 and chose the following to highlight new therapeutic choices for diabetes management. As in the past, we are not going to include much on new insulin treatment options or the artificial pancreas as these have been covered in other chapters in this article.

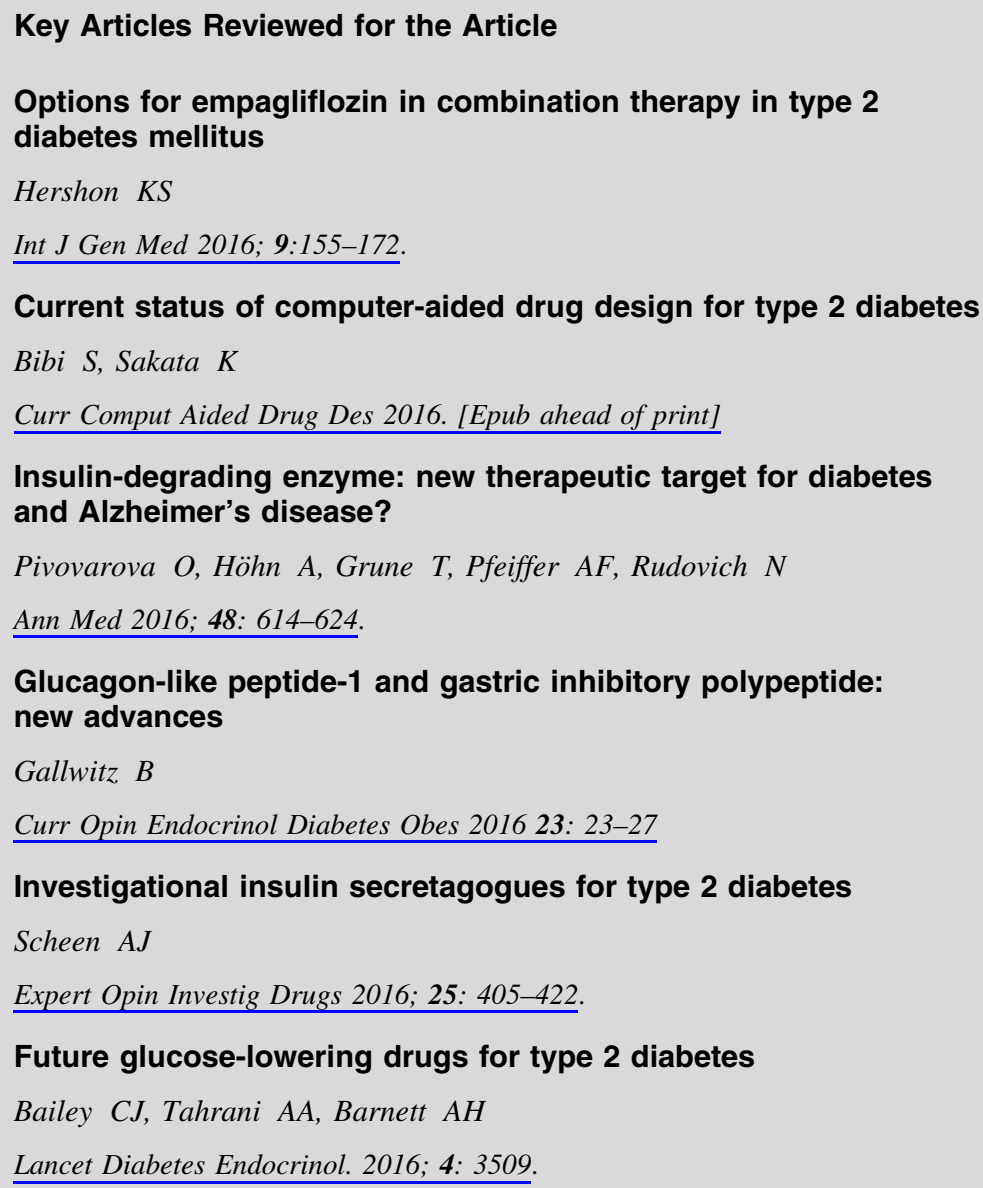


A mathematical model of the pathogenesis, prevention, and reversal of type 2 diabetes

Ha J, Satin LS, Sherman AS

Endocrinology 2016; 157: 624-635.

Identifying barriers to appropriate use of metabolic/bariatric surgery for type $\mathbf{2}$ diabetes treatment: Policy Lab results

Rubin JK, Hinrichs-Krapels S, Hesketh R, Martin A, Herman WH, Rubino $F$

Diabetes Care 2016; 39: 954-963.

Efficacy and safety of otelixizumab use in new-onset type 1 diabetes mellitus

Guglielmi C, Williams SR, Del Toro R, Pozzilli $P$

Expert Opin Biol Ther 2016;16: 841-846.

Addressing unmet medical needs in type 1 diabetes: a review of drugs under development

Mittermayer F, Caveney E, De Oliveira C, Alexander Fleming G, Gourgiotis L, Puri M, Tai LJ, Rick Turer J

Curr Diabetes Rev 2016. [Epub ahead of print]

Strategies for clinical trials in type 1 diabetes

Ehlers $M R$

J Autoimmun 2016; 71: 88-96.

Effect of metformin added to insulin on glycemic control among overweight/ obese adolescents with type 1 diabetes: a randomized clinical trial

Libman IM, Miller KM, DiMeglio LA, Bethin KE, Katz ML, Shah A, Simmons JH, Haller MJ, Raman S, Tamborlane WV, Coffey JK, Saenz AM, Beck RW, Nadeau KJ; for the T1D Exchange Clinic Network Metformin RCT Study Group

JAMA 2015; 314: $2241-2250$

A new role for an old drug: metformin targets microRNAs in treating diabetes and cancer

Zhou $J Y, X u$ B, Li L

Drug Dev Res 2015; 76: 263-269.

Continuous glucose monitoring: a consensus conference of the American Association of Clinical Endocrinologists and American College of Endocrinology

Fonseca VA, Grunberger G, Anhalt H, Bailey TS, Blevins T, Garg SK, Handelsman $Y$, Hirsch IB, Orzeck EA, Roberts VL, Tamborlane W; on behalf of the Consensus Conference Writing Committee

Endocr Pract 2016; 22: 1008-1021.

Ipragliflozin improves hepatic steatosis in obese mice and liver dysfunction in type 2 diabetic patients irrespective of body weight reduction

Komiya C, Tsuchiya K, Shiba K, Miyachi Y, Furuke S, Shimazu N,

Yamaguchi S, Kanno K, Ogawa $Y$

PLoS One 2016; 11: e0151511.

Acetyl-CoA carboxylase inhibition by ND-630 reduces hepatic steatosis, improves insulin sensitivity, and modulates dyslipidemia in rats

Harriman G, Greenwood J, Bhat S, Huang X, Wang R, Paul D, Tong L, Saha AK, Westlin WF, Kapeller R, Harwood HJ Jr

Proc Natl Acad Sci USA 2016; 113: E1796-1805. 


\author{
New therapeutic approaches in diabetic retinopathy \\ Vaziri K, Schwartz SG, Relhan N, Kishor KS, Flynn HW Jr \\ Rev Diabet Stud. 2015; 12: 196-210. \\ Diabetic macular edema: options for adjunct therapy \\ Calvo P, Abadia B, Ferreras A, Ruiz-Moreno O, Verdes G, Pablo LE \\ Drugs 2015; 75: 1461-1469.
}

Place of sulfonylureas in the management of type 2 diabetes mellitus in South Asia: a consensus statement

Kalra S, Aamir AH, Raza A, Das AK, Azad Khan AK, Shrestha D, Qureshi F, Fariduddin, Pathan F, Jawad F, Bhattarai J, Tandon $N$, Somasundaram $N$, Katulanda P, Sahay R, Dhungel S, Bajaj S, Chowdhury S, Ghosh S,

Madhu SV, Ahmed T, Bulughapitiya $U$

Indian J Endocrinol Metab 2015; 19: 577-596

Gastrointestinal microbiome modulator improves glucose tolerance in overweight and obese subjects: a randomized controlled pilot trial

Rebello CJ, Burton J, Heiman M, Greenway FL

J Diabetes Complications 2015; 29: 1272-1276.

Gut microorganisms as promising targets for the management of type 2 diabetes

Delzenne NM, Cani PD, Everard A, Neyrinck AM, Bindels $L B$

Diabetologia 2015; 58: 2206-2217.

\section{CURRENT STATUS OF SODIUM GLUCOSE CO-TRANSPORTER (SGLT) INHIBITORS IN DIABETES MANAGEMENT}

\section{Options for empagliflozin in combination therapy in type 2 diabetes mellitus}

\section{Hershon $K S^{1,2}$}

${ }^{1}$ North Shore Diabetes and Endocrine Associates, New Hyde Park, NY; ${ }^{2}$ Department of Medicine, Hofstra Northwell School of Medicine, Hempstead, NY

\section{Int J Gen Med 2016; 9: 155-172}

\section{Objective}

Present clinicians with an updated overview of empagliflozin for the treatment of type 2 diabetes mellitus (T2DM), with focus on its use in combination regimens.

\section{Methods}

Using the Medline database, keyword searches were undertaken to identify literature reporting the use of empagliflozin treatment in clinical trials with a minimum duration of 12 weeks relating to patients with T2DM.

\section{Results}

When given as monotherapy or in combination therapy (as single-pill therapy or an add-on) with sulfonylurea, metformin, linagliptin, pioglitazone, and insulin, empagliflozin was able to produce reductions that were clinically meaningful in glycated hemoglobin levels, bodyweight, plasma glucose concentrations, and blood pressure, which changes were sustained during long-term treatment. In a dedicated cardiovascular event trial, on top of standard care, empagliflozin demonstrated a significant reduction in the risk of all-cause mortality as well as cardiovascular mortality. Across the clinical trials, combination therapies with empagliflozin were well tolerated, and used alone, empagliflozin was not associated with an increased risk of hypoglycemia versus placebo. In fact, the combination of metformin and empagliflozin had a significantly reduced rate of hypoglycemia compared with the combination of metformin with a sulfonylurea. However, empagliflozin treatment did have increased risk of genital infections compared with a placebo. To date, in clinical trials diabetic ketoacidosis was not seen more frequently with empagliflozin than with placebo, although physicians should remain alert to the possible occurrence of this rare event.

\section{Conclusions}

For patients with T2DM, empagliflozin has the potential to make an important contribution to their treatment. For some patients, empagliflozin may be used as monotherapy, but its most likely use is in combination with other therapies. Given that when empagliflozin was employed in addition to standard care in patients having high cardiovascular risk there was a reduced risk of mortality, in combination with the lack of alternative options for patients with a lower cardiovascular risk, it is possible that empagliflozin will be added to ongoing regimens for a significant proportion of patients. 


\section{Comment}

The cardiovascular outcome trials (CVOTs) have recently added interesting choices for possible medical interventions while managing type 2 diabetes, although the drugs are from the same family. Specifically, an empagliflozin study was shown to have reduced overall mortality, especially from cardiovascular disease. Even though the CVOTs were criticized by many endocrinologists and the Food and Drug Administration (FDA) was blamed for making this a burden for new diabetes drug approvals, it appears that the outcome of many of these trials are guiding the providers to individualize therapy for patients with diabetes. It also appears that different medications from the families of glucagon like peptide-1 (GLP-1) analogs or SGLT2 inhibitors may be significantly different with cardiovascular outcomes or overall mortality by using these drugs in patients with type 2 diabetes. One caveat is that the population evaluated in many of the CVOTs are not identical and sometimes it is hard to extrapolate data between trials. The ideal trial/study would be a head to head comparison between different molecules from the same family of drugs in similar high risk individuals with cardiovascular disease, and evaluate the long-term outcomes in mortality and cardiovascular death. Unfortunately, such studies are unlikely to be done unless the National Institute of Health (NIH) or other non-profit organizations are willing to fund such a long-term study.

\section{NEWER INVESTIGATIONAL AGENTS}

\section{Current status of computer-aided drug design for type 2 diabetes}

Bibi S, Sakata K

Department of Environment and Life Engineering, Graduate School of Engineering, Maebashi Institute of Technology, Maebashi, Gunma, Japan

Curr Comput Aided Drug Des 2016. [Epub ahead of print]

\section{Background}

Diabetes is a metabolic disorder requiring multiple therapeutic approaches. The ability of the pancreas to produce insulin hormone properly is lost in patients with diabetes mellitus. During 2012, more than one million people across the globe died of diabetes. This was the eighth leading cause of death.

\section{Objective}

Most of the currently available drugs that are approved by the U.S. Food and Drug Administration cannot attain an adequate level of glycemic control in diabetic patients, and they have many side effects; therefore, new classes of compounds are needed. Efforts based on computer-aided drug design (CADD) are able to mine a large number of databases to produce new and potent possibilities and to minimize the requirement in terms of time and dollars for new discoveries to be made.

\section{Methods}

The pharmaceutical sciences have made advances in drug design concepts. Virtual screening of large databases is compatible with a number of computational methods including molecular docking, quantitative structure-activity relationship, pharmacophore, and molecular dynamic simulation. The contribution of these methods in the selection of antidiabetic compounds was discussed.

\section{Results}

The CADD approach has contributed to the successful discovery of novel antidiabetic agents. This mini-review focuses on the CADD approach in terms of currently approved drugs and new therapeutic agents in development that might achieve desirable glucose levels and decrease the risk of hypoglycemia, which latter is a major obstacle to glucose control and a particular concern for therapies where insulin levels are increased.

\section{Conclusion}

Drug design and development for type 2 diabetes were actively studied. However, a large number of drugs for diabetes are still in the early stages of development. The conventional target- and structure-based approaches can be viewed as a part of the efforts toward therapeutic mechanismbased drug design for the treatment of type 2 diabetes. It is expected that further improvement in the CADD approach will improve new discoveries.

\section{Comment}

Most drugs that are approved by the FDA or the European Medical Agency (EMA) have to go through a rigorous clinical trial program and, in many cases, it may include CVOT trials. These pathways are expensive and add to the cost of medications while delaying the introduction of possible therapies for patients with diabetes. Many efforts are being made in computer-aided drug design (CADD) programs that can minimize the duration of time and significantly reduce the cost for new therapeutic choices. This will require new thinking on the part of regulatory agencies where computer aided drug design programs can be used for drug approvals.

\section{Insulin-degrading enzyme: new therapeutic target for diabetes and Alzheimer's disease?}

Pivovarova $O^{1,2,3}$, Höhn $A^{3,4}$, Grune $T^{3,4,5}$, Pfeiffer $A F^{1,2,3}$, Rudovich $N^{1,2,3}$

${ }^{I}$ Department of Clinical Nutrition, German Institute of Human Nutrition Potsdam-Rehbruecke, Nuthetal, Germany; ${ }^{2}$ Department of Endocrinology, Diabetes and Nutrition, Campus Benjamin Franklin, Charite University Medicine, Berlin, Germany; ${ }^{3}$ German Center for Diabetes Research, München, Germany; ${ }^{4}$ Department of Molecular Toxicology, German Institute of Human Nutrition Potsdam-Rehbruecke, Nuthetal, Germany; ${ }^{5}$ German Center for Cardiovascular Research, Berlin, Germany

Ann Med 2016; 48: 614-624. 
Insulin-degrading is caused mainly by Insulin-degrading enzyme (IDE). It also degrades many other targets including atrial natriuretic peptide, glucagon, and betaamyloid peptide and also regulates proteasomal degradation as well as other cell functions. IDE represents a pathophysiological link between late onset Alzheimer's disease (AD) and type 2 diabetes (T2DM). Potent and selective modulators of the activity of IDE are potential drugs for therapies of both of these diseases. Acute treatment with one such novel IDE inhibitor was recently tested on mice as therapy for T2DM. In contrast, effective IDE activators can be used as treatment for AD. However, because of the pleiotropic action of IDE, sustained treatment with systemic IDE modulators should be tested carefully in animal studies. Substrate-selective IDE modulators have the potential to overcome possible adverse effects of IDE modulators that are associated with multiplicity of IDE targets. In summary, IDE represents a pathophysiological link between T2DM and AD. Some modulators of IDE activity are candidate drugs for treating both T2DM and AD. Development of substrate-selective IDE modulators could overcome possible adverse effects of IDE modulators associated with multiplicity of IDE targets.

\section{Comment}

Insulin-degrading enzyme (IDE) is mostly responsible for degrading insulin subcutaneously. In addition, IDE degrades many other target hormones like glucagon and atrial natriuretic peptide etc. Selective IDE inhibitors have been shown to positively affect Alzheimer's disease (AD). It appears that type 2 diabetes (T2D) and AD have a potential pathophysiological link. Thus, selective modulators of IDE activity are potential drugs for both AD and T2D. This is a fascinating area of research and may facilitate newer therapeutic substrate specific choices with fewer side effects.

\section{Glucagon-like peptide-1 and gastric inhibitory polypeptide: new advances}

Gallwitz $B$

Department of Medicine IV, Eberhard-Karls-University Tübingen, Tübingen, Germany

Curr Opin Endocrinol Diabetes Obes 2016; 23: 23-27

\section{Objective}

Glucagon-like peptide-1 (GLP-1) and gastric inhibitory polypeptide (GIP) are gastrointestinal peptides that both play an important role as incretin hormones in the regulation of insulin secretion and plasma glucose. Therefore GLP-1based therapies have been used as treatment for type 2 diabetes (T2D). The aim of this review is to summarize the new treatment options relating to T2D that employ GLP-1-based therapies. In addition, we also summarize relevant extrapancreatic effects that have been recently characterized for both peptides.

\section{Findings}

The review highlights novel findings that regard changes in GLP-1 secretion after bariatric surgery in which GLP-1 plays a role in promoting body weight loss and diabetes remission. For T2D therapy, novel options with long-acting GLP-1 analogs that show a desirable safety and efficacy profile are summarized, also in combination with insulin and for obesity treatment. As GIP is not suitable for T2D therapy, recent characterization of the extrapancreatic effects of GIP, mainly on bone metabolism, are described. These illustrate that the activated GIP receptor is important to allow optimal bone structure and mass.

\section{Summary}

This review summarizes new findings on the physiology and pathophysiology of GLP-1 and GIP and novel therapeutic aspects.

\section{Investigational insulin secretagogues for type 2 diabetes}

Scheen $A J^{1,2}$

${ }^{1}$ Division of Clinical Pharmacology, Center for Interdisciplinary Research on Medicines (CIRM), University of Liège, Liège, Belgium; ${ }^{2}$ Division of Diabetes, Nutrition and Metabolic Disorders, Department of Medicine, CHU, Liège, Belgium

Expert Opin Investig Drugs 2016; 25: 405-422.

\section{Introduction}

Key features in the pathophysiology of type 2 diabetes (T2D) are insulin secretory defects. Classical insulinsecreting agents, for example sulfonlyureas, stimulate insulin secretion independently of glucose and cause hypoglycemia. In spite of the advantages offered by incretin-based therapies, there remains a requirement for the development of new insulin secretagogues for the treatment of T2D.

\section{Area Covered}

This article discusses the following areas: new advances in the field of incretin-based therapies, glucokinase (GK) activators, imeglimin, free fatty acid receptor (FFAR) or G protein-coupled receptor (GPR) agonists (GPR40, GPR119, GPR120), and some other insulin secretagogues with different and wide ranging mechanisms of action that are still in preclinical development.

\section{Expert Opinion}

New insulin secretagogues should be able to offer major advantages compared to sulfonylureas and gliptins. The challenges are the avoidance of uncontrolled insulin secretion and minimization of the risk of hypoglycemia, protection of cells from a progressive loss of mass, as well as function, for a better durability of glucose control, and a good safety profile. A number of approaches are in development although it is too early to tell if one novel pharmacological class will emerge as a clinically useful insulin secretagogue in the near feature. 
Future glucose-lowering drugs for type 2 diabetes Bailey $\mathrm{CJ}^{1}$, Tahrani $A A^{2}$, Barnett $A H^{2,3}$

${ }^{I}$ School of Life and Health Sciences, Aston University, Birmingham, UK; ${ }^{2}$ Department of Diabetes and Endocrinology, Heart of England NHS Foundation Trust, Birmingham, UK; ${ }^{3}$ Centre for Endocrinology, Diabetes and Metabolism, University of Birmingham, Birmingham, $U K$

Lancet Diabetes Endocrinol. 2016; 4: 3509.

The progressive and multivariable natural history of type 2 diabetes limits how effective available glucose-lowering drugs can be. Constraints that are imposed by comorbidities (particularly cardiovascular disease and renal impairment) together with the need to avoid weight gain, hypoglycemia, and drug interactions further complicate the treatment process. Alongside research into new pharmacological entities, these challenges have prompted development of new formulations and delivery methods for existing drugs. Advances in incretin-based therapies include a miniature osmotic pump that can be implanted to give continuous delivery of a glucagonlike peptide-1 receptor agonist for 6-12 months and onceweekly tablets of dipeptidyl peptidase-4 inhibitors. Hybrid molecules that combine properties of selected incretins and other peptides are in the early stages of development, and proof of concept has been demonstrated for small nonpeptide molecules to activate glucagon-like peptide-1 receptors. Additional sodium-glucose cotransporter inhibitors are advancing through development as well as potential small-molecule inhibitors of glucagon action and novel insulin-releasing biological agents. Adiponectin receptor agonists, selective peroxisome proliferator-activated receptor modulators, cellular glucocorticoid inhibitors, and analogues of fibroblast growth factor 21 are being considered as possible new approaches to the lowering of glucose. Future opportunities for treatment have been suggested by compounds that can enhance insulin receptor and postreceptor signaling cascades or directly promote selected pathways of glucose metabolism. However, pharmacological interventions that are able to restore normal $\beta$-cell function and $\beta$-cell mass, normalize insulin action, and fully correct glucose homoeostasis are a distant vision.

\section{A mathematical model of the pathogenesis, prevention, and reversal of type 2 diabetes}

$H a J^{1}$, Satin $L S^{2}$, Sherman $A S^{1}$

${ }^{1}$ Laboratory of Biological Modeling, National Institute of Diabetes and Digestive and Kidney Diseases, National Institutes of Health, Bethesda, MD; ${ }^{2}$ Department of Pharmacology and Brehm Diabetes Research Center, University of Michigan, Ann Arbor, MI

\section{Endocrinology 2016; 157: 624-635.}

Type 2 diabetes (T2D) is generally believed to result from the combination of 2 metabolic defects, resistance to insulin, which results in an increase in the level of insulin required to maintain glucose within the normal range, and the failure of insulin-secreting pancreatic $\beta$-cells to compensate for the increased demand. We develop a mathematical model based on that pioneered by Topp and colleagues to determine how compensation succeeds or fails. Their model had an additional layer of slow negative feedback compared to the classic insulin-glucose loop in the form of a slow, glucosedependent birth and death law that governed $\beta$-cell mass. We have added regulation of 2 aspects of $\beta$-cell function on intermediate time scales to that model. The model quantifies the relative contributions of insulin secretion defects and insulin action to T2D and elucidates why prevention is easier than cure. The latter is a result of a threshold that separates the normoglycemic and diabetic states (bistability), which is also responsible for the success of bariatric surgery and acute caloric restriction in rapidly reversing T2D. The threshold concept allows for new insight into "Starling's Law of the Pancreas," whereby insulin secretion is higher for both prediabetics and early diabetics than for normal individuals.

\section{Comment}

The above four abstracts highlight different approaches to managing type 2 diabetes. Combining GLP-1 and GIP hormones might offer an advantage of managing type 2 diabetes specifically due to extra pancreatic effects of GIP, mainly on the bone metabolism.

There are many investigational insulin secretagogues for better management of type 2 diabetes that can possibly reduce the risk of hypoglycemia and may help in protecting cells in progressive loss of beta cell mass. Different secretagogues include GK activators, FFAR, GPR, GPR-40 and Imeglimin. It may be too premature to conclude if any of these are going to make for human use from what has been seen in the animal studies.

The last article highlights a mathematical model pioneered by the authors to elucidate how compensation succeeds or fails. Their threshold concept gives new insight to "Starling's Law of Pancreas" where insulin secretion is higher for subjects with prediabetes and early diabetes than for normal individuals.

\section{SURGICAL APPROACH FOR MANAGEMENT OF TYPE 2 DIABETES}

\section{Identifying barriers to appropriate use of metabolic/bariatric surgery for type 2 diabetes treatment: Policy Lab results}

Rubin $J K^{l}$, Hinrichs-Krapels $S^{l}$, Hesketh $R^{l}$, Martin $A^{2}$, Herman $W^{3}$, Rubino $F^{4}$

${ }^{I}$ The Policy Institute at King's, King's College London, London, UK; ${ }^{2} R A N D$ Europe, Cambridge, UK; ${ }^{3}$ University of Michigan, Ann Arbor, MI; ${ }^{4}$ Metabolic and Bariatric Surgery, Division of Diabetes and Nutritional Sciences, King's College London and King's College Hospital, London, UK

Diabetes Care 2016; 39: 954-963.

Despite the fact of increasing recognition of the safety, efficacy, and cost-effectiveness of bariatric/metabolic surgery in the treatment of type 2 diabetes, few potential candidates among patients who might benefit from this type of surgery avail themselves of this treatment option. To identify the practical and conceptual barriers relating to the appropriate use of surgical procedures, a Policy Lab was hosted at the third World Congress on Interventional Therapies for Type 2 Diabetes on 29 September 2015. Twenty-six stakeholders participated in the Policy 
Lab, including clinicians, policy-makers, academics industry leaders, and patient representatives. Each participant was provided with a summary of the available evidence regarding the cost-effectiveness of bariatric/metabolic surgery together with the costs related to increasing the use of bariatric/metabolic surgery using scenarios in the United States and the United Kingdom as examples of distinct systems of health-care. Among this stakeholder group there was widespread agreement that bariatric/metabolic surgery is a cost-effective and legitimate approach to treating type 2 diabetes in obese patients.

Four building blocks were identified as facilitating policy changes, these were: (1) communicating the scale of the harms and the costs that are associated with the rising prevalence of type 2 diabetes; (2) articulating well the role of bariatric/ metabolic surgery with particular population groups; (3) identifying new funding sources for bariatric/metabolic surgery; and (4) incorporating bariatric/metabolic surgery into the most appropriate clinical pathways. While more research is needed to identify appropriate clinical scenarios where bariatric/metabolic surgery might be prioritized, the case appears now to be strong enough that relevant policy-makers and practitioners should be engaged in a concerted discussion regarding how to make better use of metabolic surgical resources together with other interventions in good diabetes practice.

\section{Comment}

Metabolic/bariatric surgery for treatment of type 2 diabetes is being practiced at more centers across the globe. Therefore, it is necessary to assess the efficacy, safety, and cost effectiveness of such treatment. It is also imperative that appropriate guidelines and candidates who might benefit from such treatments be properly established. The consensus so far has been that bariatric surgery in morbidly obese people with type 2 diabetes is cost effective and is a legitimate course for management of the disease. One does not know if bariatric surgery done before the onset of type 2 diabetes may in fact completely prevent the onset of type 2 diabetes. The anecdotal data suggests that to be the case. Unfortunately, even in subjects with type 1 diabetes, significant obesity $(\mathrm{BMI}>35)$ is quite common and many such individuals may be subjected to this extreme metabolic surgery. However, such treatment in type 1 diabetes has been done only in limited number of cases across the globe. It is important to consider patient safety before implementing such invasive steps as individuals with type 1 diabetes will be on insulin for the rest of their life and are at high risk of severe hypoglycemia. In patients with T1D, special protocols may need to be devised for good outcomes after the bariatric surgery.

\section{NEWER AGENTS FOR MANAGEMENT OF TYPE 1 DIABETES}

\section{Efficacy and safety of otelixizumab use in new-onset type 1 diabetes mellitus}

\author{
Guglielmi $C^{1}$, Williams $S R^{2}$, Del Toro $R^{1}$, Pozzilli $P^{1,2}$ \\ ${ }^{1}$ Unit of Endocrinology and Diabetes, Department of Med- \\ icine, University Campus Bio-Medico di Roma, Rome, Italy;
}

${ }^{2}$ Centre of Immunology, Barts and The London School of Medicine and Dentistry, Queen Mary, University of London, London, UK

Expert Opin Biol Ther 2016; 16: 841-846.

\section{Introduction}

Type 1 diabetes (T1DM), an immune-mediated disease that is induced by antigen-specific T-cells infiltrating pancreatic beta cells, leads to a progressive loss of endogenous secretion of insulin.

\section{Areas Covered}

Specific components of the autoimmune response have been identified to favor implementation of a number of immunomodulatory therapies that include the antiCD3 monoclonal antibody (mAb) known as otelixizumab. Otelixizumab, a chimeric monoclonal antibody, targets the $\varepsilon$-chain of the CD3Tlymphocyte surface receptor that was developed to enable short therapeutic courses that are capable of inducing a state of remission of T1DM. Clinical trials have been undertaken with otelixizumab to evaluate its efficacy and safety, however, although the results of the Phase I and II studies have been positive, the results of the Phase III studies are contradictory.

\section{Expert Opinion}

High doses of otelixizumab have been shown to have beneficial effects on the beta cell function whereas a dose that is lower, tested to avoid adverse effects associated with the higher doses, was not effective for beta cells preservation. We believe that otelixizumab is a drug of possible interest in the treatment of new onset

T1DM patients and it should be considered for use in combination with other immunomodulatory agents as a solution that circumvents adverse effects while maintaining efficacy.

\section{Addressing unmet medical needs in type 1 diabetes: a review of drugs under development}

Mittermayer F, Caveney E, De Oliveira C, Alexander
Fleming G, Gourgiotis L, Puri M, Tai LJ, Rick Turner J

Quintiles GmbH, Vienna, Austria

Curr Diabetes Rev 2016. [Epub ahead of print]

The occurrence of type 1 diabetes (T1D) is increasing across the globe and there is an extremely important requirement for effective therapies. Apart from pramlintide and insulin, essentially there are no pharmacologic therapies that are currently approved for the treatment of T1D. Drugs that are already used for type 2 diabetes together with a number of new drugs, are under clinical development for T1D that include compounds that have both new and established mechanisms of action. The majority of the new compounds that are in clinical development are currently in Phases 1 and 2. In this review, the drug classes discussed include new insulins, GLP-1 agonists, SGLT inhibitors, immunomodulatory drugs including anticytokines and autoantigens, and agents that regenerate $\beta$-cells. Considerations are also provided with regards to the regulatory environment for the clinical development of T1D-related drugs, with a focus on the United States Food and Drug Administration and the 
European Medicines Agency. Future opportunities, which include combination treatments of immunomodulatory and $\beta$-cell regenerating therapies, are also discussed.

\section{Strategies for clinical trials in type 1 diabetes}

\section{Ehlers $M R$}

Clinical Trials Group, Immune Tolerance Network, San Francisco, $C A$

\section{J Autoimmun 2016; 71: 88-96.}

Over the previous decade or two, a great deal of progress has been made relating to our understanding of the immunopathology of type 1 diabetes (T1D) together with the potential of immune interventions that are able to alter the natural history of the disease. This progress is a consequence of the use of standardized endpoints, study designs, and, to some extent, mechanistic analyses relating to intervention trials in the setting of new-onset T1D. Most of these trials to date have involved single-agent interventions, however, future trials will increasingly test therapeutic combinations based on testable mechanistic hypotheses and a compelling scientific rationale. Novel trial designs will benefit these increasingly complex trials (including, for example, factorial or adaptive designs), enhanced clinical endpoints that more directly assess islet pathology (such as $\beta$-cell death assays and islet or pancreatic imaging), improved analyses of the responder, and sophisticated mechanistic assays providing deep phenotyping of lymphocyte subsets, gene expression profiling, in vitro T-cell functional assessments, and antigenspecific responses. With this developing armamentarium of enhanced trial designs, endpoints, and clinical and mechanistic response analyses, we can expect significant progress towards a better understanding of the breakdown in immunologic tolerance in T1D and how it may be restored to achieve significant and long-lasting preservation of islet function.

\section{Comment}

As indicated in the introduction, type 1 diabetes is also on the rise and thus there are many new ways to recognize the disease early. The recognition of the disease at an early stage may allow prompt immune interventions with possibly better outcomes. The above four manuscripts/abstracts highlight different strategies for clinical trials with focus on combination immune-modulation for type 1 diabetes. It's important to keep in mind that there are several regulatory challenges both in the United States by the FDA and in Europe by the EMA that limits what can or cannot be done. It is likely future options may include immune modulation along with beta cell regenerating therapies.

\section{Effect of metformin added to insulin on glycemic control among overweight/obese adolescents with type 1 diabetes: a randomized clinical trial}

Libman IM ${ }^{1}$, Miller $K M^{2}$, DiMeglio $L A^{3}$, Bethin $K E^{4}$, Katz $M L^{5}$, Shah $A^{6}$, Simmons $J^{7}{ }^{7}$, Haller $M J^{8}$, Raman $S^{9}$, Tamborlane $W V^{10}$, Coffey $J K^{11}$, Saenz $A M^{2}$, Beck $R W^{2}$, Nadeau $K^{12}$; for the T1D Exchange Clinic Network Metformin RCT Study Group
${ }^{1}$ Children's Hospital of Pittsburgh of UPMC, Pittsburgh, $P A ;{ }^{2}$ Jaeb Center for Health Research, Tampa, FL; ${ }^{3}$ Indiana University School of Medicine, Indianapolis, IN; ${ }^{4}$ University at Buffalo School of Medicine and Biomedical Sciences, Buffalo, NY; ${ }^{5}$ Joslin Diabetes Center, Boston, MA; ${ }^{6}$ Stanford University School of Medicine, Stanford, CA; ${ }^{7}$ Vanderbilt University, Nashville, TN; ${ }^{8}$ University of Florida, Gainesville, FL; ${ }^{9}$ Children's Mercy Hospital, Kansas City, MI; ${ }^{10}$ Yale University, New Haven, CT; ${ }^{11}$ University of Iowa, Iowa City, IA $;{ }^{12}$ University of Colorado, Anschutz Medical Campus, Aurora, CO

JAMA 2015; 314: 2241-2250

This article is also discussed in Article on Diabetes Technology and Therapy in the Pediatric Age Group, page

\section{Importance}

Prior studies aimed at assessing the effect of metformin on glycemic control in the adolescent population with type 1 diabetes have not produced conclusive results.

\section{Objective}

To assess the safety and efficacy of metformin in addition to insulin in treating overweight adolescents who have type 1 diabetes.

\section{Design, Setting and Participants}

A multicenter (26 pediatric endocrinology clinics), placebocontrolled, double-blind, and randomized clinical trial was designed that involved 140 adolescents with age 12.1 to 19.6 years (mean [SD] 15.3 [1.7] years), mean type 1 diabetes duration 7.0 (3.3) years, mean HbA1c $8.8 \%(0.7 \%)$, mean body mass index (BMI) 94th (4) percentile, and mean total daily insulin $1.1(0.2) \mathrm{U} / \mathrm{kg}$.

\section{Interventions}

Randomization to receive metformin $(\mathrm{n}=71)(\leq 2000 \mathrm{mg} / \mathrm{d})$ or placebo $(n=69)$.

\section{Main Outcomes and Measures}

The primary outcome was that the change in $\mathrm{HbAlc}$ from the baseline to 26 weeks adjusted for baseline HbA1c. The secondary outcomes included total daily insulin, change in blinded continuous glucose monitor indices, BMI, waist circumference, body composition, blood pressure, and lipids.

\section{Results}

Between October 2013 and February 2014, 140 participants enrolled. The baseline HbAlc was $8.8 \%$ in each group. At 13 week follow-up, the reduction in $\mathrm{HbA1c}$ was higher with metformin $(-0.2 \%)$ than with the placebo $(0.1 \%$; mean difference, $-0.3 \%$ [95\% CI, $-0.6 \%$ to $0.0 \%$ ]; $P=0.02$ ). However, this differential effect had not been sustained at the -week follow up where the mean change in $\mathrm{HbA} 1 \mathrm{c}$ from the baseline was $0.2 \%$ in each group (mean difference, $0 \%$ [95\% CI, $-0.3 \%$ to $0.3 \%$ ]; $P=0.92$ ). At 26 week follow-up, the total daily insulin measured per $\mathrm{kg}$ of body weight was reduced by a minimum of $25 \%$ from the baseline among $23 \%$ (16) of the participants in the metformin group vs. $1 \%$ (1) of 
participants in the placebo group (mean difference, $21 \%$ [95\% CI, $11 \%$ to $32 \%] ; P=0.003$ ), and $24 \%$ (17) of the participants in the metformin group and $7 \%$ (5) of the participants in the placebo group had a reduction in their BMI z score of at least $10 \%$ from the baseline to 26 weeks (mean difference, $17 \%$ [95\% CI, $5 \%$ to $29 \%$ ]; $P=0.01$ ). Gastrointestinal adverse events were reported by a greater number of participants in the metformin group than in the placebo group (mean difference, 36\% [95\% CI, 19\% to 51\%]; $P<0.001)$.

\section{Conclusions and Relevance}

Among the population of overweight adolescents with type 1 diabetes, addition of metformin to insulin did not, after 6 months, improve glycemic control. Of multiple secondary end points, findings only favored metformin for insulin dose and measures of adiposity; conversely, use of metformin did result in an increased risk for gastrointestinal adverse events. These results do not support the prescribing of metformin to adolescents who are overweight and have type 1 diabetes with the aim of improving their glycemic control.

\section{A new role for an old drug: metformin targets microRNAs in treating diabetes and cancer}

Zhou $J Y^{1}, X u B^{2}, L i L^{3}$

${ }^{1}$ Department of Anatomy and Cell Biology, McGill University, Montreal, Quebec, Canada; ${ }^{2}$ Department of Cardiology, Drum Tower Hospital, Medical School of Nanjing University, Nanjing, China; ${ }^{3}$ Department of Physician Assistant, College of Health Professions, Central Michigan University, Mount Pleasant, MI

Drug Dev Res 2015; 76: 263-269.

MicroRNAs (miRNAs) constitute a family of short, noncoding, 19-23 base pair RNA molecules. Because of their unique role in gene regulation in various tissues, miRNAs play a number of important roles in regulating insulin secretion, cancer biology, and metabolic disease. There is emerging evidence that demonstrates that miRNAs might also form novel diagnostic markers for a variety of disease states. Furthermore, it has been found that miRNAs function as either oncogenes or tumor suppressor genes in certain cancers. There has been an increasing number of studies conducted to investigate new drugs that target miRNAs as a possible anticancer therapy. Metformin is the medication that is most widely prescribed for treating type 2 diabetes (T2D) and recent clinical data suggest that metformin impacts the miRNA profile in T2D subjects. Most exciting is the fact that studies have discovered that metformin is protective against cancer. The anticancer activity of metformin is mediated through a direct regulation of miRNAs, which in addition modulates several downstream genes in metabolic or preoncogenic pathways. These miRNAs are, as such, possible therapeutic targets for treating diabetes and cancer, which subject forms the topic of this review. Further study on the regulation of miRNAs by metformin may result in novel therapeutic strategies for recurrent or drug-resistant cancer, and might form part of a combinatorial approach with conventional anticancer therapies.

\section{Comment}

Metformin has been used and promoted off-label for treatment of type 1 diabetes in conjunction with insulin therapy. Previous small studies have shown beneficial effects on reducing weight, improving glucose control, and overall reduction of insulin dose. However, this large randomized clinical trial using metformin in type 1 diabetes, did not show any improvement in glucose control after six months. There were small changes in insulin dose and measures of obesity however, with an increased risk of gastrointestinal adverse events. Thus it appears that off-label use of metformin in type 1 diabetes is not recommended.

The second abstract highlights the use of metformin in the treatment of diabetes and cancer. There are several ongoing studies that are addressing whether metformin use may improve cancer outcomes. It also needs to be determined if use of metformin might offer novel therapeutic strategies for recurrent and drug resistant cancers.

\section{CONTINUOUS GLUCOSE MONITORS FOR GLYCEMIC CONTROL}

\section{Continuous glucose monitoring: a consensus conference of the American Association of Clinical Endocrinologists and American College of Endocrinology}

Fonseca $V A^{1}$, Grunberger $G^{2}$, Anhalt $H^{3}$, Bailey $T S^{4}$, Blevins $T^{5}$, Garg $S K^{6}$, Handelsman $Y^{7}$, Hirsch $I B^{8}$, Orzeck EA', Roberts VL ${ }^{10}$, Tamborlane $W^{11}$; on behalf of the Consensus Conference Writing Committee

${ }^{1}$ Professor of Medicine and Pharmacology, Tullis Tulane Alumni Chair in Diabetes, Chief, Section of Endocrinology, Tulane University Health Sciences Center, New Orleans, LA; ${ }^{2}$ President, American Association of Clinical Endocrinologists, Chairman, Grunberger Diabetes Institute, Bloomfield Hills, MI, Clinical Professor, Internal Medicine and Molecular Medicine \& Genetics, Wayne State University School of Medicine, Detroit, MI, Professor, Internal Medicine, Oakland University William Beaumont School of Medicine, Rochester, MI, Visiting Professor, Internal Medicine, First Faculty of MI, Charles University, Prague, Czech Republic; ${ }^{3}$ Chief Medical Officer, T1D Exchange, Boston, MA; ${ }^{4}$ Director, AMCR Institute, Clinical Associate Professor, UCSD School of Medicine, San Diego, CA; ${ }^{5}$ Texas Diabetes and Endocrinology, Austin, TX; ${ }^{6}$ Professor of Medicine and Pediatrics, Director, Adult Program, Editor-in-Chief, Diabetes Technology and Therapeutics, Barbara Davis Center for Diabetes, University of Colorado Denver, Aurora, CO; ${ }^{7}$ Medical Director \& Principal Investigator, Metabolic Institute of America, Immediate Past President, American College of Endocrinology, Tarzana, CA; ${ }^{8}$ Professor of Medicine, University of Washington School of Medicine, Seattle, WA; ${ }^{9}$ Endocrinology Associates, Houston, TX; ${ }^{10}$ Professor of Internal Medicine, University of Central Florida College of Medicine, Orlando, FL; ${ }^{11}$ Professor and Chief of Pediatric Endocrinology, Yale School of Medicine, New Haven, CT

Endocr Parct 2016; 22: 1008-1021. 


\section{Objective/Methods}

The use of continuous glucose monitoring (CGM) continues to be hampered by the barriers to the adoption of this desirable technology for diabetes management. The American Association of Clinical Endocrinologists (AACE) and the American College of Endocrinology (ACE) held a public consensus conference February 20, 2016, to review the available data on CGM and to propose strategies for expanding access to CGM.

\section{Results}

It was agreed by conference participants that the available evidence supports the benefits of CGM use for type 1 diabetes (T1D) and that these benefits will probably apply whenever intensive insulin therapy is used, irrespective of the type of diabetes. CGM is likely to reduce the utilization of healthcare resources for chronic and acute complications, although real world analyses are required in order to confirm the possible cost savings and improvements in quality of life. Ongoing technological advances have improved CGM usability and accuracy, although further innovations are required in human factors, data delivery, reporting, and interpretation to foster increased use. The development of standardized data reporting using similar metrics for all devices would facilitate patient and clinician understanding and uptake of CGM. There is an urgent need for expanded CGM coverage by government and private payers.

\section{Conclusion}

CGM improves glycemic control, reduces hypoglycemia, and could possibly reduce overall costs associated to diabetes management. Expanding the coverage and use of CGM may improve health outcomes for people with diabetes.

\section{Comment}

I chose this abstract/manuscript to be included in this chapter not because I am a coauthor but due to its importance in the management of diabetes. The AACE consensus conference on CGM highlighted the role of CGM in reducing hypoglycemia, improving glucose control, and possible reduced health care costs. Despite these benefits, as has been previously reported from large databases like T1D exchange, the full potential of this new technology is not implemented in the majority of the patients. The data shows that both patients with type 1 and type 2 diabetes benefit from using these technologies. In fact, on July 21, 2016, the FDA panel voted in favor that CGM data could be used nonadjunctively to adjust insulin dose. The reasons for lower uptake of such technology in management of diabetes may be due to limited resources and educational needs required to implement CGM in clinical practice. The authors strongly recommended that a simple technology like CGM should be implemented in larger patient populations. Hopefully this will happen in the United States, especially if the Center for Medicare and Medicaid Services (CMS) starts covering the cost of CGM for patients with diabetes.

\section{NEWER THERAPIES FOR HEPATIC STEATOSIS IN PATIENTS WITH DIABETES}

\section{Ipragliflozin improves hepatic steatosis in obese mice and liver dysfunction in type 2 diabetic patients irrespective of body weight reduction}

Komiya $C^{l}$, Tsuchiya $K^{l}$, Shiba $K^{l}$, Miyachi $Y^{l}$, Furuke $S^{l}$, Shimazu $N^{l}$, Yamaguchi $S^{l}$, Kanno $K^{2}$, Ogawa $Y^{1,3}$

${ }^{I}$ Department of Molecular Endocrinology and Metabolism, Graduate School of Medical and Dental Sciences, Tokyo Medical and Dental University, Tokyo, Japan; ${ }^{2}$ Kanno Clinic, Tokyo, Japan; ${ }^{3}$ Japan Agency for Medical Research and Development, CREST, Tokyo, Japan

PLoS One 2016; 11: e0151511.

Type 2 diabetes mellitus (T2DM) is associated with a high incidence of nonalcoholic fatty liver disease (NAFLD) that is related to insulin resistance and obesity. At the moment, medical interventions for NAFLD focus on exercise and diet control in order to reduce body weight, and there is a need for effective pharmacological therapies. Sodium-glucose cotransporter 2 (SGLT2) inhibitors are oral antidiabetic drugs that promote urinary excretion of glucose by preventing its reabsorption into the renal proximal tubules. SGLT2 inhibitors lower the blood glucose levels independently of insulin action and it is expected that they should reduce body weight because of urinary calorie loss. Here, we illustrate that the SGLT2 inhibitor ipragliflozin improves hepatic steatosis in high-fat, diet-induced, and leptin-deficient (ob/ob) obese mice independently of weight loss. In obese mice, ipragliflozininduced hyperphagia increased energy intake, acting to attenuate body weight reduction with increased epididymal fat mass. An inverse correlation exists between the weights of the liver and epididymal fat in ipragliflozin-treated obese mice, which suggests that ipragliflozin treatment promotes normotopic fat accumulation in the epididymal fat and prevents ectopic fat accumulation in the liver. Despite increased adiposity, ipragliflozin ameliorates obesity-associated inflammation and insulin resistance in epididymal fat. Clinically, ipragliflozin enhances liver dysfunction in patients with T2DM independently of weight loss. These findings provide new insight into the effects of SGLT2 inhibitors on energy homeostasis and fat accumulation and also indicate their potential therapeutic efficacy in T2DM-associated hepatic steatosis.

\section{Acetyl-CoA carboxylase inhibition by ND-630 reduces hepatic steatosis, improves insulin sensitivity, and modulates dyslipidemia in rats}

Harriman $G^{l}$, Greenwood $J^{2}$, Bhat $S^{b}$, Huang $X^{3}$, Wang $R^{d}$, Paul $D^{4}$, Tong $L^{4}$, Saha $A K^{5}$, Westlin $W F^{l}$, Kapeller $R^{l}$, Harwood $H J{ }^{1}$

${ }^{I}$ Nimbus Therapeutics, Cambridge, MA; ${ }^{2}$ Schrodinger Inc., New York, NY; ${ }^{3}$ Pharmaron Beijing Co. Ltd., Beijing, China; ${ }^{4}$ Department of Biological Sciences, Columbia University, New York, NY; ${ }^{5}$ Department of Medicine and Physiology, School of Medicine, Endocrinology, Diabetes, and Nutrition, Boston University, Boston, MA

Proc Natl Acad Sci USA 2016; 113: E1796-1805. 
Simultaneous inhibition of the acetyl-CoA carboxylase (ACC) isozymes $\mathrm{ACC} 1$ and $\mathrm{ACC} 2$ results in the stimulation of fatty acid oxidation and concomitant inhibition of fatty acid synthesis and might favorably affect the mortality and morbidity and associated with diabetes, obesity, and fatty liver disease. We use structure-based drug design to identify a series of strong allosteric protein-protein interaction inhibitors. These are exemplified by ND-630, which interacts within the ACC phosphopeptide acceptor and the dimerization site to prevent dimerization and to inhibit the enzymatic activity of both ACC isozymes, reduce fatty acid synthesis and to stimulate fatty acid oxidation in animals and cultured cells, and that exhibits desirable drug-like properties. When administered chronically to rats that have diet-induced obesity, ND-630 reduces hepatic steatosis, improves insulin sensitivity, favorably affects dyslipidemia, and reduces weight gain without affecting food intake. When administered chronically to Zucker diabetic fatty rats, ND-630 acts to improve glucosestimulated insulin secretion, reduce hepatic steatosis, and to reduce hemoglobin A1c (with a $0.9 \%$ reduction). These data together suggest that ACC inhibition by representatives of this series might be useful in the treatment of a variety of metabolic disorders, including metabolic syndrome, fatty liver disease, and type 2 diabetes mellitus.

\section{Comment}

Hepatic steatosis is a major risk factor for patients with type 2 diabetes and is closely associated with being overweight and obese. Many estimates point to the fact that nonalcoholic steatohepatitis (NASH)/nonalcoholic fatty liver disease (NAFLD) may become the leading cause for liver transplant in the future. It's known that if the liver fat exceeds $6 \%$ to $10 \%$ the patient is likely to have insulin resistance irrespective of type 1 or type 2 diabetes. Many new therapeutic options are being evaluated for possible treatment for hepatic steatosis. Use of SGLT-2 inhibitor reduce hepatic steatosis and promote normal fat accumulation in the epididymal fat. Other ways of treating hepatic steatosis may include simultaneous inhibition of acetyl-CoA carboxylase (ACC) isozymes ACC1 and ACC2. Such studies in animals have suggested a significant reduction in A1c along with decreasing incidence of fatty liver disease.

\section{THERAPUTIC APPROACHES FOR TREATMENT OF DIABETIC RETINOPATHY}

\section{New therapeutic approaches in diabetic retinopathy}

Vaziri $K^{1}$, Schwartz $S G^{1}$, Relhan $N^{1}$, Kishor KS ${ }^{1}$, Flynn $H W \mathrm{Jr}^{l}$

${ }^{I}$ Department of Ophthalmology, Bascom Palmer Eye Institute, University of Miami Miller School of Medicine, Miami, FL

Rev Diabet Stud. 2015; 12: 196-210.

Diabetic retinopathy is a common microvascular complication of diabetes mellitus and a large proportion of adults over the age of 40 in the United States are affected by it. The condition is one of the leading causes of visual loss. A great deal of attention has been given to increasing the role of current treatments together with investigating a number of new therapies and drug delivery methods. In the treatment of diabetic macular edema (DME), intravitreal pharmacotherapies, especially antivascular endothelial growth factor (anti-VEGF) agents, have gained popularity. At the moment, anti-VEGF agents are often used as the first-line agents in center-involved DME, and recent data have suggested that among these agents, aflibercept leads to better visual outcomes in patients with worse baseline visual acuities. Although photocoagulation remains the standard treatment for proliferative diabetic retinopathy (PDR), the recent FDA approvals of aflibercept and ranibizumab for the management of diabetic retinopathy associated with DME might indicate potential for pharmacologic treatments of PDR. Novel therapies, including chemokines, small interfering RNAs, kallikrein-kinin inhibitors, and various antiangiogenic agents, are currently being evaluated for use in the management of both diabetic retinopathy and DME. In addition, novel drug delivery methods such as sustained-release implants and refillable reservoir implants are either under active evaluation or have recently gained FDA approval. This review gives an update on new developments in the treatment of diabetic retinopathy.

\section{Diabetic macular edema: options for adjunct therapy}

Calvo $P^{1,2}$, Abadia $B^{l}$, Ferreras $A^{1,2}$, Ruiz-Moreno $O^{1,2}$, Verdes $G^{3}$, Pablo $L E^{1,2}$

${ }^{I}$ Department of Ophthalmology, Miguel Servet University Hospital, Aragon Health Science Institute, IIS Aragon, Zaragoza, Spain; ${ }^{2}$ University of Zaragoza, Spain: ${ }^{3}$ Endocrinology Department, Obispo Polanco Hospital, Teruel, Spain

\section{Drugs 2015; 75: 1461-1469.}

Diabetes mellitus (DM) is a chronic disease that affects 387 million people across the globe. Diabetic retinopathy (DR), which is a common complication of DM, is the main cause of blindness in the active population. Diabetic macular edema (DME) can occur at any stage of DR and it is characterized by vascular hyperpermeability together with hard exudates within the macula. Timely intervention through surgical and medical therapies have reduced the risk of severe vision loss occurring by more than 90\%. During 2012, intravitreal ranibizumab became the first antivascular endothelial growth factor (anti-VEGF) agent approved for DME and subsequently many reports relating to the use of ranibizumab for DME have been shown to be promising. Randomized, prospective, multicenter clinical trials, in particular RESOLVE, READ-2, RISE/RIDE, RESTORE, DRCR.net protocol I, and RETAIN have reported improvements related to best-corrected visual acuity and decreased central retinal thickness measured with optical coherence tomography in patients with DME. Clinical trials evaluating intravitreal aflibercept and bevacizumab (DAVINCI, VISTA/VIVID, and BOLT) and more recently DRCR.net protocol $\mathrm{T}$ have noted similar treatment benefits. Intravitreal steroids (dexamethasone intravitreal implant and fluocinolone acetonide), particularly in refractory cases, also play an important role in management of DME (MEAD/CHAMPLAIN and FAMOUS/FAME 
studies). In summary, during the past five years, blocking VEGF and inflammation has been demonstrated to improve visual outcomes in patients with macular edema because of DM that has revolutionized the treatment of center-involved DME and has established a new standard of care.

\section{Comment}

We have not given much attention to changes in longterm diabetes micro- and macrovascular complications over the past three decades. It is well known that incidence rates for diabetic retinopathy and macular edema have significantly decreased ( $\sim 10 \%$ now) with better understanding and management strategies for diabetes care. However, due to a global increase (pandemic) in the number of patients with diabetes and suboptimal care in $50 \%$ of patients with diabetes, the actual number of patients with microvascular complications has risen.

Several studies have now shown beneficial effects in using intraocular injections of anti-VEGF agents for treatment of macular edema and proliferative diabetic retinopathy. Many agents have now received FDA approval for this use. In addition to these anti-VEGF agents, intravitreal steroid implants, especially in refractory cases, have played a significant role in macular edema and improvement in vision.

\section{Place of sulfonylureas in the management of type 2 diabetes mellitus in South Asia: a consensus statement}

Kalra $S^{1}$, Aamir $A H^{2}$, Raza $A^{3}$, Das $A K^{4}$, Azad Khan $A K^{5}$, Shrestha $D^{6}$, Qureshi $F^{7}$, Fariduddin ${ }^{8}$, Pathan $F^{9}$, Jawad $F^{10}$, Bhattarai $J^{11}$, Tandon $N^{12}$, Somasundaram $N^{13}$, Katulanda $P^{14}$, Sahay $R^{15}$, Dhungel $S^{16}$, Bajaj $S^{17}$, Chowdhury $S^{18}$, Ghosh $S^{19}$, Madhu $S V^{20}$, Ahmed $T^{21}$, Bulughapitiya $U^{22}$

${ }^{I}$ Department of Endocrinology, Bharti Hospital and BRIDE, Karnal, Haryana, India; ${ }^{2}$ Department of Endocrinology, Post Graduate Medical Institute Hayatabad Medical Complex, Peshawar, Pakistan; ${ }^{3}$ Shaukat Khanum Memorial Cancer Hospital and Research Centre, Lahore, Pakistan; ${ }^{4}$ Department of Endocrinology, Pondicherry Institute of Medical Sciences, Puducherry, India; ${ }^{5}$ Department of Public Health, Bangladesh University of Health Sciences, Dhaka, Bangladesh; ${ }^{6}$ Department of Endocrinology, Norvic International Hospital, Kathmandu, Nepal; ${ }^{7}$ Department of Endocrinology, Al-Khaliq Medicare Hospital, Dhaka, Bangladesh; ${ }^{8}$ Department of Endocrinology, Bangabandhu Sheikh Mujib Medical University, Shahbag, Dhaka, Bangladesh; ${ }^{9}$ Department of Endocrinology, BIRDEM Hospital, Dhaka, Bangladesh; ${ }^{10}$ Department of Diabetology, Medilink Clinics, Karachi, Pakistan; ${ }^{11}$ Department of Medicine, Trivuvan University, Kathmandu, Nepal; ${ }^{12}$ Department of Endocrinology and Metabolism, All India Institute of Medical Sciences, Ansari Nagar, New Delhi, India; ${ }^{13}$ South Asian Federation of Endocrine Societies, National Hospital, Dhaka, Bangladesh; ${ }^{14}$ Department of Clinical Medicines, Diabetes Research Unit, University of Colombo, Colombo, Sri Lanka; ${ }^{15}$ Department of Endocrinology, Osmania Medical College, Hyderabad, Telangana, India; ${ }^{16}$ Department of
Medicine, Nepal Medical College Teaching Hospital, Kathmandu, Nepal; ${ }^{17}$ Department of Medicine, MLN Medical College, Allahabad, Uttar Pradesh, India; ${ }^{18}$ Department of Endocrinology, IPGMER and SSKM Hospital, Kolkata, West Bengal, India; ${ }^{19}$ Department of Endocrinology and Metabolism, IPGMER, Kolkata, West Bengal, India; ${ }^{20}$ Department of Medicine and Head, Centre for Diabetes, Endocrinology and Metabolism, UCMS-GTB Hospital, New Delhi, India; ${ }^{21}$ Department of Endocrinology, BIRDEM, Dhaka, Bangladesh; ${ }^{22}$ Department of Endocrinology, Kalubowila South Teaching Hospital, Kalubowila, Sri Lanka

Indian J Endocrinol Metab 2015; 19: 577-596.

Since sulfonylureas (SUs) were introduced into clinical practice during the 1950 s they have remained the main-stay of pharmacotherapy in the management of type 2 diabetes. Despite benefits that are well-established, their place in therapy is being unjustifiably overshadowed by newer therapies. Many of the clinical issues associated with the use of SUs are agent-specific and as such do not pertain to the class. Modern SUs (glimepiride, gliclazide MR) are supported by a large body of experience, evidence, and most importantly, outcome data, which support their role in managing patients with diabetes. Person-centered care, i.e., a careful choice of SU, appropriate dosage, adequate patient counseling, and timing of administration will ensure that deserving patients do not miss out on the advantages of this well-established class of antidiabetic agents. Considering their safety, efficacy pleiotropic benefits, and the low cost of therapy, SUs should be considered as a recommended therapy for the treatment of diabetes in South Asia. This initiative by SAFES has the aim of encouraging the safe, rational, and smart prescription of SUs, and includes appropriate medication counseling.

\section{Comment}

With all the new therapeutic choices for management of type 2 diabetes, sulfonylureas (SUs) have become the target for not using them in clinical practice. For the past 50 years, SUs have been used in the management of type 2 diabetes. In late 1960, University Group Diabetes Program (UGDP) study raised the concern of cardiovascular safety with the use of SUs. However, their clinical utility is well documented despite the known side effect of hypoglycemia, especially when longer acting SUs are used. One wonders if some of this criticism of SUs is driven by the industry, who sees the benefit of using newer therapies to their bottom line. The SUs are cheap and effective and are still used frequently in the developed world (United States) and in the developing world, and for now should remain a possible option for management of type 2 diabetes.

\section{RECENT ADVANCES IN GUT MICROBIOMES AS POTENTIAL THERAPUTIC TARGETS}

\section{Gastrointestinal microbiome modulator improves glucose tolerance in overweight and obese subjects: a randomized controlled pilot trial}

Rebello $\mathrm{CJ}^{1,2}$, Burton $\mathrm{J}^{1}$, Heiman $M^{3}$, Greenway $F L^{1}$ 
${ }^{1}$ Pennington Biomedical Research Center, Louisiana State University System, Baton Rouge, LA; ${ }^{2}$ School of Nutrition and Food Sciences, Louisiana State University, Baton Rouge, LA; ${ }^{3}$ MicroBiome Therapeutics LLC, New Orleans, LA

J Diabetes Complications 2015; 29: 1272-1276.

\section{Objective}

The aim of this study was to examine the effects of a gastrointestinal microbiome modulator (GIMM) containing blueberry anthocyanins, inulin, $\beta$-glucan, and blueberry polyphenols on satiety, metabolic parameters, and fecal markers of gut microbiota.

\section{Design and Methods}

Thirty obese or overweight participants with an age of between 18 and 70 years, enrolled in a randomized controlled trial. The participants consumed either the placebo or the test product daily for four weeks. Stool samples were collected and blood was drawn at baseline and week four for the assessment of glucose control, gut microbiota, satiety hormones and lipid measures. Subjective satiety was examined weekly. Linear models were used to compare differences from baseline to week four.

\section{Results}

The consumption of GIMM resulted in an improvement of blood glucose tolerance $(P=0.008)$ and also increased satiety $(P=0.03)$. There were no statistically significant differences in plasma satiety hormones, insulin sensitivity, fecal markers of gut microbiota, or serum lipid concentrations between the two groups. However, the plasma satiety hormones and fecal short chain fatty acid concentrations were increased in the test group compared to the placebo group.

\section{Consultation}

GIMM consumption for four weeks increases satiety and improves glucose tolerance, possibly through insulinindependent pathways.

\section{Gut microorganisms as promising targets for the management of type 2 diabetes}

Delzenne $N M^{l}$, Cani $P D^{1,2}$, Everard $A^{l}$, Neyrinck $A M^{l}$, Bindels $L B^{1}$

${ }^{1}$ Metabolism and Nutrition Research Group, Louvain Drug Research Institute, Université catholique de Louvain, Brussels, Belgium; ${ }^{2}$ Walloon Excellence in Life sciences and BIOtechnology (WELBIO), Louvain Drug Research Institute, Université catholique de Louvain, Brussels, Belgium

Diabetologia 2015; 58: 2206-2217.

Every human intestine harbors not only hundreds of trillions of bacteria but also viruses, bacteriophage particles, fungi, and archaea, which make up a dynamic and complex ecosystem that is referred to as the gut microbiota. Over the past 10 years, an increasing amount of data have indicated changes in gut bacterial function or composition in type 2 diabetic patients. Analysis of this "dysbiosis" allows for the detection of alterations in specific bacteria, clusters of bacteria, or bacterial functions that are associated with the occurrence or evolution of type 2 diabetes; these bacteria are mainly involved in the control of inflammation and energy homeostasis. Our review is focused on two key questions: does gut dysbiosis actually play a role in the occurrence of type 2 diabetes, and will recent discoveries linking the gut microbiota to host health be helpful for the development of new therapeutic approaches for type 2 diabetes? Here we review how surgical, pharmacological, and nutritional interventions for type 2 diabetic patients could impact gut microbiota. Experimental studies in animals are identifying which bacterial metabolites and components act on host immune homeostasis and glucose metabolism, largely by targeting the intestinal cells involved in endocrine and gut barrier functions. We discuss novel approaches (e.g., probiotics, prebiotics, and fecal transfer) together with the need for research and adequate intervention studies to determine the feasibility and relevance of these novel therapies for the management of type 2 diabetes.

\section{Comment}

Gastrointestinal (GI) tract and various GI hormones play an important role in diabetes e.g., incretins, gastric inhibitory peptide (GIP), and pancreatic polypeptide (PP) etc. The gut microbiome has become the recent buzz word for satiety, overweight and obesity, and type 2 diabetes. Many studies have shown that fecal transplant and other gut microbiota might help in better understanding obesity and type 2 diabetes. This is an emerging area, but needs further work to see its possible role in day to day management of the disease.

\section{Summary and Conclusions}

It is fair to assume that criticism of CVOT trials mandated by the FDA for non-insulin therapeutic drugs for type 2 diabetes was not well liked by the industry and the diabetologists. It is also evident that all molecules are not the same even though they belong to the same family. The best way to manage diabetes may be an individualized approach where a given molecule may be better for a particular subject with diabetes. As highlighted above, to reduce the cost of medication and expedite the approval of therapeutic choices for people with diabetes, computer-aided programs (CADD) may be necessary for early and safe introduction of newer therapies. Lastly, we need to work together to reduce the ever increasing burden of type 2 diabetes globally; whether by lifestyle modification or other appropriate therapies. If the prevalence of diabetes continues to increase, it will consume the majority of the rising cost of health-care.

\section{Author Disclosure Statement}

The authors for this chapter, S. K. G., D. G., T. G., and V. N. S. do not have any disclosures. 